\title{
EL AUGE DEL MOVIMIENTO DE EDUCACIÓN ALTERNATIVA, ANTECEDENTES, CARACTERÍSTICAS Y REFLEXIONES SOBRE SU FUTURO
}

\section{THE RISE OF THE ALTERNATIVE EDUCACTION MOVEMENT. BACKGROUND, CHARACTERISTICIS AND REFLECTIONS ON YOUR FUTURE}

\author{
Aitor Garagarza', \\ Israel Alonso ${ }^{2}$ \\ Miren Aguirregoitia ${ }^{3}$ \\ Universidad del Pais Vasco/ Euskal Herriko \\ Unibertsitatea
}

\section{ABSTRACT}

Los movimientos de educación alternativa se han convertido en los últimos años en tendencia y ha crecido el número de propuestas educativas que se enmarcan dentro de este

1 https://orcid.org/0000-0002-2019-6085 -https:// scholar.google.com/citations?user=ITdSdRoAAAAJ\&hl=es\& $o i=a$

Universidad del Pais Vasco/ Euskal Herriko Unibertsitate. Aitor Garagarza aigara3@hotmail.com

2 https://orcid.org/0000-0003-1139-5223 -https:// scholar.google.es/citations?user=SIUs7D8AAAAJ\&hl=es\&oi =ao

Universidad del Pais Vasco/ Euskal Herriko Unibertsitatea ISRAEL ALONSO israel.alonso@ehu.eus

3 https://orcid.org/0000-0001-7372-2459Universidad del Pais Vasco/ Euskal Herriko Unibertsitatea movimiento a nivel del Estado Español y mundial. Aquí presentamos la revisión de la literatura realizada en torno al concepto de educación alternativa aportando una definición propia de esta e identificando las características generales comunes a este movimiento. Para realizar este análisis, ha sido necesario revisar los antecedentes clave de este movimiento de educación alternativa dado que, aunque sus proyectos se presenten en ocasiones como alternativas innovadoras, son pedagogías y metodologías basadas en propuestas de hace más de un siglo como son las de Alexander Sutherland Neill o Rebeca y Mauricio Wild. Para ello, se han anali- 
zado además de las pedagogías antecesoras, las experiencias educativas de Summerhill (Reino Unido), El León Dormido (Ecuador) y Paideia (España). Los resultados de la revisión realizada muestran que la educación alternativa es un concepto donde se integran diferentes teorías, cosmovisiones y realidades, pero del cual también se recogen características comunes de estos proyectos que ayudan a entender las razones por las cuáles en pleno siglo XXI estas propuestas han alcanzado el auge e importancia del que hablamos.

\section{INTRODUCCIÓN}

En los últimos años, ante la incertidumbre que nos plantea el sistema sociopolítico y económico global, la nueva sociedad compleja y diversa, y las voces críticas que ponen en entredicho la función de la escuela y la educación convencional, son múltiples los proyectos que se están desarrollando en diferentes partes del planeta bajo el concepto de "educación alternativa". El crecimiento de este movimiento ha sido exponencial en los últimos años (García, 2017).

Este crecimiento nos dirige hacia el análisis y revisión del recorrido de este movimiento, su concepción, las características pedagógicas y metodológicas que se encuentran bajo el paraguas de "educación alternativa", sus antecedentes y su situación actual. El análisis de estos factores nos lleva a afirmar que estos planteamientos pedagógicos a pesar de no ser nuevos en el tiempo, están siendo capaces de hacer frente a los retos educativos del presente $y$ del futuro, y cabe preguntarse si la escuela convencional empezará a asumir, parcialmente al menos, estas propuestas ya que, se puede afirmar que el movimiento de educación alternativa cada vez es más sólido y está más presente en el imaginario colectivo de la sociedad como propuesta educativa válida.

Este artículo se basa en una investigación realizada sobre el movimiento de educación alternativa y su relación con los con- ceptos de ciudad, comunidad y el desarrollo educativo. Desde esta perspectiva, se presenta en un primer momento una revisión bibliográfica sobre los debates en torno al concepto de educación alternativa, sus características y criterios comunes. Teniendo en cuenta que existen diferentes percepciones al respecto y gran variedad de planteamientos y metodologías se ha optado por aportar una concepción heterodoxa y se han buscado aquellas características comunes que comparten diferentes tendencias que trabajan dentro de la innovación educativa.

Posteriormente, se ha indagado en sus orígenes, en aquellas experiencias que fueron pioneras y han servido de marco de referencia para otros proyectos. Hablamos de Summerhill en Gran Bretaña, La Pesta en Ecuador o Paideia en el Estado Español. Acto seguido aportamos un esbozo sobre la situación actual que nos sirve para visibilizar el auge (Larrañeta, 2015) de espacios educativos alternativos, su futuro y la posibilidad de que podamos estar viviendo una nueva ola educativa.

\section{CONCEPTO Y CARACTERÍSTICAS}

Adentrarnos dentro del campo de la educación alternativa nos lleva inexorablemente a analizar y sintetizar el término. Los matices, las cosmovisiones, las realidades y los diferentes contextos. Lo que hoy se entiende por alternativo, puede que en otro espacio y tiempo no lo sea. Es más, lo alternativo puede tener vocación hegemónica, pretendiendo en el caso de la educación disputar y ofrecer otro modelo educativo al convencional. En cambio, si no tiene vocación hegemónica estaríamos hablando de proyectos con vocación residual.

Intentar conceptualizar qué entendemos por educación alternativa es una tarea ardua, más aún cuando el concepto es muy amplio, y puede llevar a diversos significados según las subjetividades de las personas o de los diferentes paradigmas educativos. No existe literatura que logre un denominador común sobre este 
tipo de proyectos educativos. Diferentes autores (Graubard, 1981; Contreras 2004, 2010; García, 2005; Feito, 2006; Domènech, 2009; Mateu, 2011; Pericacho, 2014; Carbonell, 2015; García, 2017; Carneros, 2018) han intentado clasificar y definir las características que cumple el movimiento de educación alternativa. Todos ellos coinciden en que es un movimiento heterogéneo y la simple catalogación de este movimiento resulta complicada: escuelas libres, educación libre, educación activa, pedagogías vivas, escuelas no convencionales, innovación educativa... son algunos de los diferentes términos que se pueden encontrar en la literatura científica de este ámbito. Es difícil, por lo tanto, hallar una definición precisa y concreta que agrupe diferentes tendencias en este ámbito. Ramos y Pericacho (2013) y Raywid (1994), parten de la misma idea, añaden que, en el campo de la educación alternativa, no solo existe diversidad en cuanto a la terminología utilizada, sino que también subsisten una gran variedad de planteamientos, métodos $y$, por consiguiente, praxis con resultados muy diferentes. Algunos, incluso afirma que, en cualquier caso, "cada tipo de alternativa conlleva una opción de carácter filosófico y político" (CEDEFOP, 2002, p.145).

El término "alternativa" en educación, pese a que se utiliza de forma constante, es un concepto muy amplio sobre el que no se ha profundizado a nivel académico y no tiene una clara definición. A falta de un significado preciso, el término "educación alternativa" describe diferentes enfoques educativos con un plan de estudios especial e innovador (Carneros, 2018, p. 88)

Carbonell (2015), presenta aquellas pedagogías alternativas que están implementando innovaciones educativas. Propuestas pedagógicas diferentes, que en muchos casos comparten parcialmente metodologías y análisis del contexto, pero que difieren a veces en la praxis o en el ámbito de actuación para la transformación educativa. En concreto, presenta 8 propuestas pedagógicas diferentes:

- Las pedagogías no institucionales. Aprendizaje y educación fuera de la escuela.

- Las pedagogías críticas.

- Las pedagogías libres no directivas. Alternativas a la escolarización ordinaria.

- Las pedagogías de la inclusión y la cooperación.

- La pedagogía lenta, serena y sostenible.

- La pedagogía sistémica.

- Las pedagogías del conocimiento integrado. Los proyectos de trabajo.

- Las pedagogías de las diversas inteligencias.

Aunque este autor (2015) hable de pedagogías del siglo XXI, en la mayoría de casos nos presenta propuestas educativas, de lo que él es consciente, que han teorizado y se han implementado durante el siglo XX. Estas propuestas cuentan con un amplio bagaje teórico, pero su implementación hasta la fecha, cuantitativamente, ha sido minoritaria. Algunas de estas propuestas en estos momentos están en auge o presentan oportunidades de manera alternativa e innovadora ante los desafíos educativos del presente (Larrañeta, 2015).

Tal como señala Carneros (2018) es importante diferenciar dentro de la educación alternativa, entre aquellos proyectos educativos alternativos y el concepto pedagogía alternativa. Según este autor, las pedagogías alternativas son aquellas que se pueden desarrollar tanto dentro de la educación convencional como en proyectos de corte alternativo. Estaríamos hablando de una metodología y no de una institución. En este caso podríamos situar experiencias referentes en nuestro entorno como Amara Berri (Guerrero, 2013) o las Comunidades 
de Aprendizaje (Cadena-Chala y OrcasitasGarcía, 2016).

Cuando hablamos de educación alternativa estamos hablando de lo que Carbonell (2015) denomina "Las pedagogías libres no directivas. Alternativas a la escolarización ordinaria" (p.93), aun así, cabe destacar la influencia de otras propuestas pedagógicas. Por ejemplo, la corriente pedagógica crítica está muy presente en el análisis de la situación educativa actual y con la cual compartimos en este trabajo su retrospectiva del papel que juega la escuela como institución reproductora de los poderes fácticos. Por otro lado, también tenemos en cuenta aquellas experiencias de "pedagogías no institucionales" (Carbonell, p.19), como "la ciudad educadora" (p.38), "la ciudad de los niños" (p.40) de Francesco Tonucci $(1996,2014)$ o las pedagogías lentas, que, si bien Carbonell las separa de las pedagogías libres, tienen sinergias claras con propuestas de esta índole.

Anteriormente hemos comentado que las prácticas educativas que se están desarrollando en muchos casos comparten metodologías. Es más, dentro de la educación alternativa, encontramos diferentes tendencias o metodologías educativas que responden a diversas preocupaciones y concepciones pedagógicas. La práctica educativa que desarrollan este tipo de proyectos es muy heterogénea entre sí. Contreras (2004, 2010) a la hora de sintetizar qué se entiende por educación alternativa, parte de aquellos aspectos del modelo escolar convencional que pretende subvertir: currículum establecido, los procesos de evaluación-calificación, la enseñanza como única vía al aprendizaje, la agrupación por edades, el control de las personas adultas en la gestión-decisión de los procesos de enseñanza, la delimitación espacial o el control de los tiempos.

Carneros (2018) sitúa dichos proyectos educativos en la búsqueda de la justicia social y ambiental, convirtiéndose en agentes para el cambio, "a través de una educación ambiental, crítica, democrática y equitativa que le permita ser consciente y combatir las injusticias del sistema (violencia, marginación, carencia de poder, explotación, imperialismo cultural e insostenibilidad, entre otras)" (p.20). Unido con los expresado realiza una definición amplia de los proyectos de educación alternativa:

proyectos que acogen a niños/as de diferentes familias bajo una estructura organizativa (cuotas, tiempos, responsables, espacio específico...), siempre y cuando se organicen siguiendo su propio criterio y voluntad, sin partir de la tradición, siendo originales, innovadoras e inconformistas en todas sus formas y por tanto no asentándose en los hábitos ni formas mayoritarias. (Carneros, 2018, p. 20)

Diferentes autores (Contreras 2004, 2010; Greenberg, 2003; García, 2005; Salmerón, 2010; Carbonell, 2015; García, 2017; Carneros, 2018) hacen referencia a elementos comunes como la libertad, respeto, autonomía, empoderamiento, creatividad y participación protagónica. Elementos que se repiten de manera diferente, pero que más allá de delimitar o crear una definición hermética y dogmática de la educación alternativa, proponen un marco interpretativo amplio, plural y capaz de agrupar las diferentes tendencias que componen el Movimiento de Educación Alternativa. También, queremos señalar la matización que realiza Carneros (2018) sobre las escuelas alternativas con modelos cerrados y las escuelas alternativas con modelos abiertos. En este artículo hacemos referencia principalmente a las segundas. 
Figura 1: Escuelas alternativas

\begin{tabular}{|c|c|c|c|}
\hline \multirow{3}{*}{$\begin{array}{l}\text { Escuelas } \\
\text { alternativas }\end{array}$} & $\begin{array}{c}\text { Escuelas infantiles } \\
\text { alternativas }\end{array}$ & \multicolumn{2}{|c|}{$\begin{array}{l}\text { Bosques-escuelas } \\
\text { Escuelas Reggio Emilia } \\
\text { Otras (Montessori, Waldorf, Libres...) }\end{array}$} \\
\hline & \multirow[t]{2}{*}{$\begin{array}{c}\text { Escuela alternativa } \\
\text { (etapa de escolari- } \\
\text { zación) }\end{array}$} & $\begin{array}{l}\text { Escuela alternativa con } \\
\text { modelos cerrados }\end{array}$ & $\begin{array}{l}\text { Escuelas Montessori } \\
\text { Escuelas Waldorf o Steiner } \\
\text { Escuelas logosóficas } \\
\text { Escuelas Dalton } \\
\text { Escuelas Jenaplan } \\
\text { Escuelas Freinet }\end{array}$ \\
\hline & & $\begin{array}{l}\text { Escuela alternativa con } \\
\text { modelos abiertos }\end{array}$ & $\begin{array}{l}\text { Escuelas libres } \\
\text { Escuelas democráticas } \\
\text { Escuelas libertarias } \\
\text { Escuelas activas } \\
\text { Escuelas respetuosas }\end{array}$ \\
\hline
\end{tabular}

Fuente: Carneros y Murillo (2017, p.133)

A continuación, partiendo de la literatura anterior pasamos a desvelar las características generales de este movimiento educativo: (a) nuevas formas de intervención para la transformación, (b) educación en libertad y libertad de elección, (c) educación holística, (d) diversidad espacial, (e) el personal profesional como acompañante, (f) agrupaciones heterogéneas, (g) espacios de aprendizaje no masificados, (h) precariedad legal y económica y (i) autogestión y participación.

Nuevas formas de intervención para la transformación

El tránsito de la modernidad sólida a la modernidad líquida (Bauman, 2013) también ha traído consigo el desvanecimiento de los grandes relatos de la izquierda revolucionaria. La apuesta hegemónica de la izquierda revolu- cionaria durante el siglo pasado, la toma del poder para la transformación de la sociedad, y sus posteriores desenlaces propicia un cambio significativo en la cultura política de cierta parte de la sociedad que se situaba sociológicamente bajo estos parámetros. Ante la irrupción del capitalismo neoliberal globalizado y el fin de la historia propugnada por Fukuyama (1989), desde diferentes sectores se ha optado por "el actual local, piensa global" que reivindicaba el movimiento antiglobalización.

Traducido al mundo de la educación, diferentes grupos de personas están desarrollando proyectos educativos alternativos, buscando nuevos modelos comunitarios, nuevas formas de relacionarse, de trabajar el desarrollo personal, las relaciones de poder... buscando desde la educación y trabajando con diversos 
actores, nuevas prácticas de intervención para el cambio (García, 2017). Unido al fracaso "del sistema educativo para afrontar los cambios, necesidades y demandas del siglo XXI y una crisis del sistema económico, político y social que ha impulsado a muchas familias y educadores a buscar respuestas colectivas que respondan a sus necesidades" (Carneros, 2018, p. 130).

\section{EDUCACIÓN EN LIBERTAD Y LIBERTAD DE ELECCIÓN}

Una de las máximas de los proyectos de educación alternativa se basa en respetar los ritmos de cada persona, teniendo en cuenta que los procesos educativos no son lineales y que cada cual debe seguir su propio camino. "La educación libre pretende ser una relación educativa basada en el respeto a la infancia y en concreto a sus necesidades" (Salmerón, 2010, p.6). Se generan ambientes en los cuales se procura que las niñas y niños sean conscientes de las decisiones que toman y sus consecuencias. En ese tránsito el interés y el deseo son claves.

Este es el mantra, la seña de identidad más emblemática. Libertad que se asocia a la conquista del bienestar y de la felicidad; a que cada cual pueda elegir su propio camino, sus propias decisiones y la formación de su propia singularidad. Ello requiere un escrupuloso y sostenido cuidado, comprensión, amor y respeto hacia la infancia por parte de las personas adultas. Una libertad que exige límites de sentido común relacionados con la seguridad y la libertad de los otros y que nada tiene que ver con el libertinaje de que cada uno hace lo que quiere, sin normas de ningún tipo. (Carbonell, 2015, p. 101).

Esta idea rompe con la visión del Curriculum Educativo como plan secuencial y homogéneo (Greenberg, 2003; Contreras, 2004, 2010). Los proyectos de educación alternativa en su mayoría no trabajan bajo ningún currículum preestablecido, los niños y niñas mediante su libre elección construyen conocimiento y se forman como personas.
En este tipo de modelos educativos no existen asignaturas obligatorias como en la escuela convencional. Otra de las premisas principales es que el aprendizaje es un ejercicio natural (Greenberg, 2003; Salmerón, 2010), planteando que si se fuerza a las personas puede conseguirse el efecto contrario. Por ello, se defiende que aprender es un acto de libertad, donde cada persona atiende a sus ritmos, intereses, curiosidades o necesidades. Cada persona decide y tiene un rol activo sobre lo que debe aprender, cuánto, cuándo, cómo y con quién (Carbonell, 2015). Es decir, se parte de la plena confianza en las niñas y niños.

Dentro de esta pedagogía existen diferentes corrientes que implementan metodologías diversas, pero por norma general la asistencia no es obligatoria. Este tipo de modelos educativos normalmente disponen de un gran abanico de ambientes y recursos, donde las niñas y niños pueden optar por la elección de sus propios itinerarios. Deciden, como hemos comentado, cuánto tiempo le dedican, de qué manera, con quién, para qué... Por lo tanto, no existe currículum previo, este se genera mediante la acción diaria (Salmerón, 2010).

\section{EDUCACIÓN HOLÍSTICA}

Además de que cada cual pueda elegir que aprender, no solo se trabaja el lado cognitivo de las personas, aspecto que es prioritario en las escuelas convencionales. Los ambientes que se generan; naturales, sociales y culturales, pretenden que se trabajen otras dimensiones; emocionales, sentimentales, relacionales, artísticas, abstractas.... impulsando el aprendizaje holístico. Es decir, "que la educación no es incorporar una cultura, sino incorporarse a una cultura, esto es, la relación que se crea entre lo personal-singular que cada uno trae al mundo, con el mundo social, natural y cultural" (Contreras, 2004, p.15). 


\section{DIVERSIDAD ESPACIAL}

La composición espacial de estos espacios dista mucho de la escuela convencional, que por norma general está dividida en aulas. En cambio, los espacios de educación alternativa suelen ser espacios sin aulas, abiertos, sin muros, donde se pueden encontrar todo tipo de ambientes, talleres, espacios polivalentes, espacios para el descanso, la investigación, la lectura, el juego... (Carbonell, 2015) donde poder desarrollar de manera multidimensional los aspectos comentados anteriormente.

El espacio exterior tiene la misma importancia que el espacio interior. En muchos casos estos proyectos suelen estar situados en zonas rurales y cuenta con sus propios espacios exteriores o simplemente se adaptan a los espacios naturales para desarrollar su actividad (García, 2017). Véase las escuelas bosque, modelo innovador que convierte a la naturaleza en aula. Los niños y niñas no acuden a ningún espacio cerrado, la naturaleza (bosque, campo o playa) se convierte en su entorno de aprendizaje (Nixon, 2015; Etxebarria, 2016).

Estas experiencias intentan crear ambientes educativos que tengan en cuenta el respeto profundo por la infancia y la juventud, adaptándolas a sus necesidades, entendiendo que estas personas son sujetos protagónicos en el entorno que se recrean; creando espacios que favorezcan la capacidad de movimiento, libertad, libre expresión, observación, escucha... (Contreras, 2004, 2010).

\section{EL PERSONAL PROFESIONAL COMO ACOMPAÑANTE}

En este modelo educativo la palabra profesor (personal profesional) o bien no se utiliza, se omite o no está muy bien vista. La labor del personal profesional se basa en el acompañamiento no directivo, la escucha activa y la intermediación, en caso de que lo soliciten, para mediar o facilitar. Las personas que trabajan en el centro intentan no intervenir en el proceso aprendizaje de las personas más pequeñas, para que ellas puedan desarrollarse, investigar, aprender, sentir, experimentar... siguiendo los principios que comentábamos anteriormente. (Carbonell, 2015; García, 2017).

\section{AGRUPACIONES HETEROGÉNEAS}

No existen ni horarios, ni asignaturas, ni niveles como en la escuela convencional. En el caso de los horarios son más flexibles, además, en muchos casos la asistencia no es obligatoria. En estos modelos educativos las personas de diferentes edades conviven y se desarrollan conjuntamente. Conviven personas de diferentes edades posibilitando la trasmisión intergeneracional de saberes (Contreras, 2004, 2010). Lo cual no quita que los ambientes que se generan tengan en consideración las diferentes etapas en el desarrollo de las personas. También asumen la heterogeneidad de las personas. Es decir, el simple hecho de asumir que personas de una misma edad biológica puedan tener ritmos y necesidades totalmente diversas.

\section{ESPACIOS DE APRENDIZAJE NO MASIFI- CADOS}

Critican la masificación de la escuela convencional, dado que no se puede atender las necesidades de todas las personas de manera individualizada y deshumaniza a las personas. Por lo tanto, se busca que sean espacios de aprendizaje no masificados, posibilitando la acción individual o grupal. El número de personas que participan normalmente no sobrepasa las dos cifras (Contreras, 2004, 2010; Salmerón, 2010; Carbonell, 2017;). Si bien, las trabas legales y económicas pueden influir en la afluencia de personas a estos proyectos (Rodríguez, 2014).

\section{PRECARIEDAD LEGAL Y ECONÓMICA}

Estos proyectos normalmente empiezan en la etapa de educación infantil. En el caso del Estado Español se debe a que hasta la etapa de educación primaria la asistencia no es obligato- 
ria, por lo tanto, las trabas legales son menores. Aun así, cada vez se están desarrollando más experiencias en educación primaria y secundaria. En estas dos etapas educativas las dificultades legales y administrativas con las que se encuentran son mayores (Rodríguez, 2014; Carneros, 2018).

Este tipo de proyectos tiene dificultades para encontrar un encaje legal. En algunos países de Europa, como Alemania, Reino Unido o Dinamarca (Nixon, 2015; Lysklett, 2017; Antonietti, 2018), la legislación reconoce este tipo de centros, incluso pueden percibir ayudas públicas. Es el caso de las escuelas bosque, por ejemplo, cuentan con el reconocimiento legal. Pero por norma general, "son escuelas que se ubican fuera del sistema educativo" (Salmerón, 2010, p.6). Estos proyectos que se encuentran en los márgenes de la ley, siendo su situación alegal, dependen normalmente de las cuotas de las familias. La subsistencia económica en la mayoría de los casos es un tanto precaria (Rodríguez, 2014; Carbonell, 2017; Carneros, 2018).

\section{AUTOGESTIÓN Y PARTICIPACIÓN}

La comunidad educativa en este modelo, suele ser sujeto activo y protagónico. La implicación de los tres actores principales que participan (familias, educadores, niñas y niños) por norma general es alta. Para ello establecen mecanismos de cohesión y participación interna donde sean escuchadas y tenidas en cuenta todas las voces, desde las personas más adultas a las de menor edad. El poder decisorio de los diferentes actores varía según el proyecto pero se intentan implementar modelos de democracia directa, participativa y horizontal. Normalmente, estos proyectos de educación alternativa se rigen por los principios de autodeterminación, autorregulación, negociación compartida, participación y colaboración (García, 2005; Salmerón; 2010, Carbonell, 2015; García, 2017; Carneros, 2018).
Figura 2: Educación Alternativa características

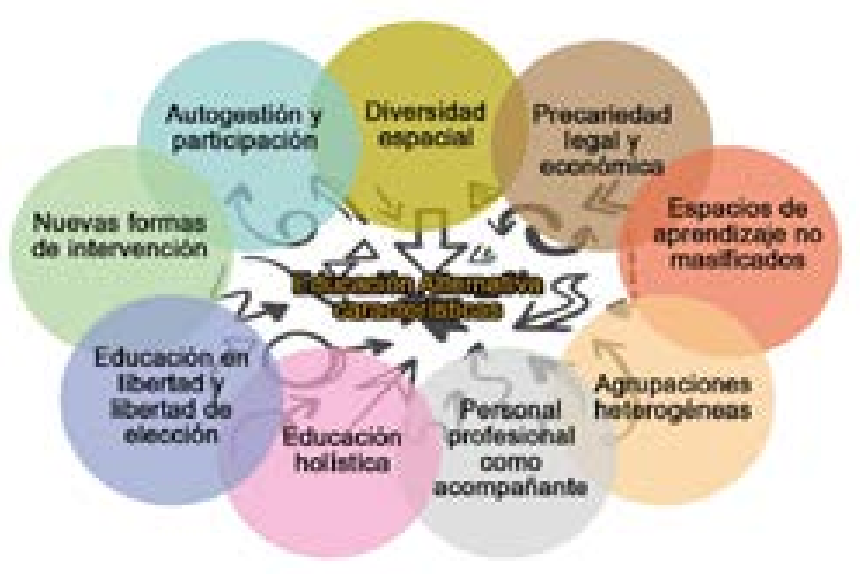

Fuente: Elaboración propia

Teniendo en cuenta las características y lo anteriormente mencionado realizamos una definición propia de educación alternativa:

son iniciativas diversas, con diferentes planteamientos filosóficos, políticos y pedagógicos, que bien fuera del sistema escolar o dentro de él, plantean formas de acción educativas no convencionales para el cambio social, que buscan la libertad individual y colectiva de las personas, impulsando el aprendizaje holístico y la participación protagónica en diversos espacios autogestionados.

Cabe aclarar, que hablamos de educación alternativa y no de escuelas alternativas, porque entendemos que, en muchos casos, este tipo de proyectos transcienden el marco espacial de la escuela. Por ello, nos parece más acertado hablar de proyectos de educación alternativa y no de escuelas alternativas.

\section{ANTECEDENTES EN EL ESTADO ESPA- ÑOL Y A NIVEL INTERNACIONAL}

Aunque este tipo de propuestas educativas hayan cobrado vigencia y estén en auge en pleno siglo XXI (Carbonell, 2015; Larrañeta, 2015; Carneros 2018), no es menos cierto, como hemos comentado anteriormente, que este tipo de propuestas se llevan desarrollando desde principios del siglo XX. 
Desde que existen instituciones escolares siempre ha habido personas y colectivos que, desde sensibilidades diferentes, han planteado alternativas educativas al modelo hegemónico. La historia de la pedagogía está repleta de personas y experiencias educativas que han sido capaces de mostrarnos una mirada más amplia (Mateu, 2011). Hoy en día la educación alternativa está de moda (...), y parece que se está buscando que represente el intento de encontrar el sentido de la educación en el siglo XXI. (Carneros, 2018, p. 132)

Vanguardia de este movimiento y de gran influencia han sido los proyectos impulsados en Gran Bretaña, a principios del siglo XX, por Alexander Sutherland Neill, Summerhill (Neill y Torner, 1968; Neill, 2009), o en Ecuador por Rebeca y Mauricio Wild, a finales del siglo $X X$. En el caso de los Wild, en un principio fue "El Pesta" y posteriormente "El León Dormido" (Wild, 2013).

No cabe hablar de los proyectos de educación alternativa sin mencionar el caso de Summerhill. Este proyecto, pionero, empezó su andadura en 1921 en Dresde (Alemania). En los años posteriores se trasladaría a diferentes localidades y en 1927 se ubicará finalmente en Leiston (Inglaterra). Fundada por Alexandre Sutherland Neill y hoy en día dirigida por sus descendientes, este proyecto educativo no ha estado exento de persecuciones legales a lo largo de su historia, que finalmente han logrado soslayar (Neill, 2009).

En la década de los 60 un editor de EEUU se puso en contacto con Neill y Torner, quienes publicaron "Summerhill: un punto de vista radical sobre la educación de los niños" (1963). Libro que logró gran éxito, aumentando la fama y repercusión a nivel internacional de este proyecto. El prestigioso intelectual, de alcance mundial, Erich Fromm (en Neill, 1963) en el prólogo de dicho libro escribiría lo siguiente:
Es un punto de vista radical sobre la crianza de los niños. En mi opinión, su libro es de gran importancia porque representa el verdadero principio de la educación sin miedo. En la Escuela Summerhill la autoridad no disfraza un sistema de manipulaciones. (p.11)

Este proyecto educativo fuertemente influenciado por las ideas del psicoanalista Wilhelm Reich busca que la educación se desarrolle en libertad. Si bien existen normas y limites, estas están consensuadas por los órganos decisorios, la asamblea, a la que pueden acudir todas las personas que participan en Summerhill. En este proyecto participan personas de diferentes edades, que conviven y se educan en los mismos espacios. Existen clases ordinales, pero nadie está obligado a asistir a ellas y cada cual debe decidir cuándo acudir. Además de las asignaturas clásicas de la escuela convencional se le da gran importancia a las actividades artísticas y creativas (Neill, 1963, 2009; García, 2017).

Rebeca y Mauricio Wild, décadas más tarde, en 1977, fundarán en Ecuador "El Pesta". Proyecto que funcionará hasta 2005, llegando incluso a las 150 alumnas y alumnos. Posteriormente, fundarán "El León Dormido". Tanto El Pesta como El León Dormido se basan en la libertad de las personas en la toma de decisiones, libertad no entendida sin límites. Esta concepción de la libertad implica crear entornos adecuados a las necesidades de las niñas y niños, posibilitando una convivencia armónica, asegurando la seguridad emocional y afectiva. Desde este planteamiento solo creando ambientes adecuados la libertad y los límites convivirán con coherencia (Nuin, 2013; Wild, 2013).

Este proyecto educativo que se divide en dos etapas de 2 a 7 (preoperativa) y de 7 a 14 años (operativa), en comparación con Summerhill no cuenta con asignaturas definidas. Este proyecto intenta generar ambientes para la libre manipulación, indagación, experimentación y formación de las personas. Ambientes de libre 
uso y disfrute según los intereses de cada persona en cada momento. Para ello existen diferentes espacios, con ambientes diversos, que nada tiene que ver con las aulas de las escuelas convencionales. En ellos podrán encontrar todo tipo de materiales. Cabe destacar, que al equipo docente se le desprende todo tipo de poder, su labor es de simple acompañamiento y en caso de que las personas más pequeñas se lo soliciten, resolver alguna duda (Nuin, 2013; Wild, 2013; García, 2017).

En los últimos años de funcionamiento de El Pesta, Rebeca Wild fue cuestionando el modelo educativo que habían construido. Observaban con preocupación cómo los padres y las madres delegaban la educación o el funcionamiento del centro en el personal profesional. "Wild ve aquí la principal fuente de conflictos entre adultos y niños, ya que los primeros viven bajo la presión de un horario inflexible, mientras que los segundos carecen de sentido del tiempo" (García, 2017, p.95). Es por ello, que en el nuevo proyecto educativo que están desarrollando está muy arraigada la idea de aprender en comunidad, compartiendo la crianza, el espacio, el tiempo y la economía (Wild, 2013).

Otra experiencia de referencia, pionera en el Estado Español es la Escuela Libre Paideia. Esta experiencia de larga trayectoria, creada en 1978, está situada en Extremadura y se define como proyecto netamente de ideología libertaria (Martín, 2004, 2006; Carneros, 2018). En Paideia huyen de toda neutralidad que se le puede otorgar a la educación, creen en "la ética libertaria del apoyo mutuo, la libertad y la igualdad para avanzar hacia una transformación global de la sociedad. Siempre desde un alto grado de compromiso político por parte de los educadores" (Carbonell, 2015, p.110). Otro aspecto fundamental de Paideia son los órganos decisorios, en este caso, las asambleas donde participan todas las personas y se toman todo tipo de decisiones. Aquí también no existen clases magistrales, cada miembro es libre de elegir cómo, con quién y qué aprender(Martín, 2004, 2006).

Existen otro tipo de proyectos con menos trayectoria, como La Caseta o El Roure en Cataluña. Estas dos experiencias surgen en el año 2000 y ambas participan de la Xarxa d'Educació Lliure. Esta Red tiene entre otros objetivos "ayudar a los proyectos de educación libre (espacios de aprendizaje) a nacer y crecer y facilitar el intercambio entre ellos" (Xarxa d'Educació Lliure, 2018).

En las últimas décadas desde diferentes corrientes pedagógicas se vienen desarrollando un sin fin de proyectos sobre todo en Europa y América Latina. Experiencias que, dependiendo de las singularidades legales de cada Estado, se encuentran en situación dispar. En muchos casos son proyectos que no cuentan con reconocimiento legal y situándose en los márgenes de la ley (Carbonell, 2015).

Otra de las peculiaridades del movimiento, heterogéneo, de educación alternativa es que está empezando a trabajar en red, construyen foros de debate y de intercambio de experiencias, aprovechando los cambios que se han producido en las telecomunicaciones y las oportunidades que ofrece la era digital. Muestra de ello es la película-documental, impulsada por la Red de Educación Alternativa (REEVO), "La educación prohibida". Película-documental financiada colectivamente, de libre distribución, y producidas, más allá de los métodos convencionales, utilizando herramientas que ofrece el Software Libre que cuenta con más de 15.000.000 de reproducciones ${ }^{4}$ en la red ${ }^{5} \mathrm{La}$ Red de Educación Alternativa que opera tan solo en América Latina, aparte de la producción

\footnotetext{
$4 \quad$ A fecha de 19 de septiembre de 2019.

$5 \quad$ A partir de estas reflexiones críticas han surgido, a lo largo de los años, propuestas y prácticas que pensaron y piensan la educación de una forma diferente. "La Educación Prohibida" es una película documental que propone recuperar muchas de ellas, explorar sus ideas y visibilizar aquellas experiencias que se han atrevido a cambiar las estructuras del modelo educativo de la escuela tradicional.
} 
de la película-documental que señalamos, también ha creado un directorio donde construyen, de forma colaborativa, un mapa con las diferentes experiencias que se están desarrollando alrededor del globo terráqueo. A fecha de 19 de septiembre de 2019 este directorio ha recogido un total 1646 experiencias, la mayoría de ellas se están desarrollando en América Latina y en el Estado Español. . En su mayoría, las propuestas educativas con las que nos encontramos reúnen las características nombradas anteriormente. Son proyectos en los cuales se impulsa la educación holística, activa, libre, participativa y democrática (Wernicke, 1994; Carbonell, 2015; García, 2017).

En el Estado Español, en 2013, Almudena García empezó a desarrollar el Directorio Ludus de Pedagogías Alternativas, contabilizando un total de 30 proyectos. Para el año 2016 había contabilizado un total de 800 proyectos enmarcados dentro de la pedagogía alternativa (García, 2017), muestra del rápido desarrollo e interés creciente que está surgiendo en torno a otros modelos educativos. Un debate que cada vez ocupa más espacio en la agenda mediática, académica y social.

No todos los proyectos que recoge el directorio Ludus (2018) se enmarcan dentro lo que anteriormente hemos caracterizado como Movimiento de Educación Alternativa, pero más de $2 / 3$ de los proyectos que recoge se sitúan en esa línea. A fecha de 17 de mayo de 2018 había contabilizado 823 proyectos; de los cuales 294 se denominan pedagógicamente escuelas libres o activas, 101 vinculadas a la modelo Montessori, 16 a las escuelas bosques y 229 a otro tipo de pedagogías.

\section{CONCLUSIONES}

A lo largo del artículo se ha podido constatar que conocer y analizar el movimiento de educación alternativa resulta indispensable hoy en día ya que cada vez está más presente en la agenda mediática, educativa, social y aca- démica. Así mismo, Es un hecho que existe muy poca literatura científica (Carneros, 2018) en torno a este movimiento y que resulta urgente dedicar más tiempo y recursos a la investigación en torno al tema.

Desde este artículo se ha constatado que intentar conceptualizar qué entendemos por educación alternativa es una tarea difícil, más aún cuando el concepto es muy amplio, y puede llevar a diversos significados según las subjetividades de las personas o de los diferentes paradigmas educativos. En la literatura analizada se ha observado que bajo el denominador "escuela alternativa" se pueden hallar muchas escuelas que presentan alternativas a la llamada escuela tradicional, y que responden a distintas concepciones y preocupaciones pedagógicas.

Coincidimos con diferentes autores (Graubard, 1981; Contreras 2004, 2010; García, 2005; Feito, 2006; Domènech, 2009; Mateu, 2011; Pericacho, 2014; Carbonell, 2015; García, 2017; Carneros, 2018) que han intentado clasificar y definir las características que cumple el movimiento de educación alternativa, al plantear que es un movimiento heterogéneo y la simple catalogación de este movimiento resulta complicada. Como explica Carneros (2018), "el término "alternativa" en educación, pese a que se utiliza de forma constante, es un concepto muy amplio sobre el que no se ha profundizado a nivel académico y no tiene una clara definición" ( $p .88)$.

En este artículo, después de un estudio riguroso de bibliografía especializada y el análisis de diferentes experiencias educativas se han presentado las características generales de este movimiento educativo. Uno de los aspectos comunes a este movimiento es que presentan nuevas formas de intervención para la transformación, es decir, diferentes grupos de personas están desarrollando proyectos educativos alternativos, buscando nuevos modelos comunitarios, nuevas formas de relacionarse y de trabajar el desarrollo personal, buscando desde la educación y trabajando con diversos actores, nue- 
vas prácticas de intervención para el cambio.

Por otro lado, coinciden en que apuestan por una educación en libertad y de libertad de elección: una de las máximas en estos proyectos es el respeto a los ritmos de cada persona, asumiendo que cada proceso educativo es único y que, por ello, no trabajan basándose en un currículum preestablecido, sino que las niñas y los niños mediante su libre elección construyen su aprendizaje y se forman como personas. En relación con este punto, desde la educación alternativa se trabaja de forma holística, es decir, no solo se centran en el desarrollo cognitivo, sino que trabajan otros aspectos de las personas entendiendo al ser humano como un todo. Para lograr este tipo de educación la educación alternativa cuestiona el rol tradicional del o la profesora y lo define como personal profesional acompañante, es decir, su labor en estos proyectos se basa en el acompañamiento no directivo, la escucha activa y la intermediación.

Además de estas características más relacionadas con la forma de entender la educación y la pedagogía existen aspectos relacionados con la organización escolar como la diversidad espacial, las agrupaciones heterogéneas o la defensa de espacios de aprendizaje no masificados que son también comunes a estos proyectos. Generalmente en estas propuestas encontraremos espacios abiertos, sin muros, donde al espacio exterior se le da la misma importancia que al interior. Por otro lado, defienden modelos educativos donde personas de diferentes edades conviven y se desarrollan de forma conjunta, son espacios donde la diversidad en todos los sentidos se convierte en un elemento que fortalece el aprendizaje. Para posibilitar esto, es necesario crear espacios que faciliten tanto la acción individual como la grupal huyendo de la masificación de las aulas convencionales.

Por último, encontramos dos aspectos de gestión que caracterizan a las propuestas alternativas y son, por un lado, la precariedad legal y económica y, por otro lado, la autogestión y participación. En relación al primer aspecto, este tipo de proyectos tiene dificultades para encontrar un encaje legal y dependen normalmente de las cuotas de las familias. La subsistencia económica en la mayoría de los casos es precaria. En cuanto a la segunda característica detectada, la comunidad educativa en este modelo, suele ser sujeto activo y protagónico. La implicación de los tres actores principales que participan (familias, educadores/as, niñas y niños) por norma general es alta. Para ello establecen mecanismos de cohesión y participación interna donde sean escuchadas y tenidas en cuenta todas las voces.

Por lo tanto, se ha podido constatar que a pesar de la gran amalgama de proyectos educativos alternativos existentes manejando diferentes pedagogías y metodologías, tienen en su mayoría elementos en común que las hace parte de un movimiento único que es la llamada educación alternativa.

Como se ha podido apreciar en el desarrollo del artículo, los discursos y las prácticas de estos proyectos de educación alternativa no es reciente ni del siglo $X X I$, sino que tienen antecedentes en los siglos que nos preceden. Pioneros fueron León Tolstoi, Paul Robín o Ferrer i Guardia (Giacomoni, 2008). Posteriormente surgieron experiencias como Summerhill, La Pesta o Paideia. Experiencias que se vienen desarrollando desde el siglo $X X$, que en estos últimos años han aumentado cuantitativamente y que en muchos casos se siguen moviendo en los márgenes de la ley. Mediante el análisis de estas experiencias se ha podido comprobar en este trabajo como las hoy llamadas escuelas alternativas se basan principalmente en estas experiencias y en muchas otras, como decía Mateu (2011) "la historia de la pedagogía está repleta de personas y experiencias educativas que han sido capaces de mostrarnos una mirada más amplia" o como explica Carbonell (2015) cuando habla de las pedagogías del siglo 
$\mathrm{XXI}$, en la mayoría de los casos son propuestas educativas que se teorizaron e implementaron durante el siglo XX.

Como exponíamos al principio de este trabajo, estas escuelas han empezado a tomar relevancia no solo socialmente, sino que académicamente deberían tener más hueco como objeto de estudio ya que han dejado de estar en una situación residual (cualitativamente) a cobrar mayor importancia y a considerarse sus prácticas pedagógicas como elementos innovadores que dan respuesta a los vacíos que la escuela convencional deja al no responder a las necesidades humanas y sociales que crea el nuevo orden mundial en la era de la globalización. Ante este sistema, prima una educación en valores, abierta y libre que cuestione las relaciones de poder, las nuevas formas de relación social y el individualismo latente, donde se vuelva a poner a la niña y el niño en el centro junto con la participación activa de toda la comunidad en la escuela y viceversa.

Podemos afirmar por tanto que la repercusión que está teniendo este movimiento educativo va creciendo continuamente. En los últimos años el crecimiento ha sido exponencial, podríamos decir que estamos ante una nueva ola educativa. Aunque cuantitativamente estemos hablando de datos muy poco significativos en comparación con la escuela convencional, estamos seguros de que la tendencia en los próximos años de este movimiento educativo de alcance global puede generar grietas y seguir inmiscuyéndose en la agenda educativa.

Es por ello, que nos parece importante seguir de cerca el recorrido de este movimiento. La evolución pedagógica de sus propuestas, las singularidades que emanan de ella, la construcción de alianzas a nivel social, los posibles movimientos institucionales, la construcción de una identidad propia...indagar en las fortalezas y debilidades de estas propuestas. Es decir, observar cómo estas iniciativas hacen frente a los retos educativos del presente.
Entendemos que son proyectos inacabados, son propuestas educativas integrales que están en proceso de construcción continua. Su propia idiosincrasia las convierte en proyectos en construcción y aprendizaje constante. Quizás esta sea una de sus fortalezas ante un mundo cambiante. La perspectiva de reinventarse y autoanalizarse continuamente, les otorga esa flexibilidad necesaria para hacer frente a los retos del futuro.

Además, la participación es una de las premisas, en la cual las familias, las personas de menor edad y sus profesionales son sujetos protagónicos. Sujetos protagónicos en las decisiones diarias, de las reflexiones de las orientaciones pedagógicas que se asumen... personas que participan activamente en la construcción de los proyectos y, por lo tanto, son parte indispensable en la socialización del movimiento educativo.

Puede que los debates pedagógicos que plantean transciendan al movimiento $\mathrm{y}$ en un momento en el cual se cuestiona la institución escolar, sus propuestas sean asumidas de manera parcial por la escuela convencional. Es decir, en los últimos tiempos estamos observando cómo propuestas de innovación educativa, algunas de ellas desde lo que denominamos educación alternativa, se están empezando a implementar en las escuelas convencionales.

Pero no nos olvidemos y pequemos de optimistas, en muchos casos son propuestas que cuentan con un amplio bagaje a sus espaldas, pero durante años han estado sumidas en el ostracismo pedagógico. Aunque el contexto sea propicio y el desarrollo de las telecomunicaciones favorezca su socialización, nada nos asegura que esto no vuelva a suceder. ¿Conseguirá el Movimiento de Educación Alternativa disputar la hegemonía a la escuela convencional? ¿O tan siquiera situarse en posiciones cuantitativamente no residuales?. 


\section{REFERENCIAS BIBLIOGRÁFICAS}

Antonietti, M. (2018). European preschools in nature. Principles and evidence in international references. Form@ re-Open Journal per la formazione in rete,18(1), 357-367.

Bauman, Z. (2002). Modernidad líquida. México D.F, México: Fondo de Cultura Económica.

- (2007). Miedo Líquido. Barcelona, Cataluña: Paidós.

Cadena-Chala, M. C., y Orcasitas-García, J. R. (2016). Comunidades de aprendizaje en el País Vasco: caracterización y organización escolar. Educación y educadores, 19(3), 373-391

Carbonell, J. (2015). Pedagogías del siglo XXI: alternativas para la innovación educativa. Barcelona, Cataluña: Octaedro.

Carneros, S. (2018). La escuela alternativa: Un modelo de búsqueda de la justicia social y ambiental (Tesis Doctoral). Universidad Autonoma de Madrid, Madrid, España.

Carneros, S., y Murillo, F. J. (2017). Aportaciones de las escuelas alternativas a la justicia social y ambiental: Autoconcepto, autoestima y respeto. REICE. Revista Iberoamericana sobre Calidad, Eficacia y Cambio en Educación,15(3), 129-150.

Centro Europeo para el Desarrollo de la Formación Profesional (CEDEFOP). (2002). Ágora IX. Modelos alternativos de formación. Luxemburgo: Oficina de Publicaciones Oficiales de las Comunidades Europeas.

Contreras, J. (2004). Una educación diferente. Cuadernos de Pedagogía, 341, 12-17.

—. (2010). Otras escuelas, otra educación, otra forma de pensar en el currículum. En Gimeno Sacristán, J., Saberes e incertidumbres sobre el currrículum. Madrid, España: Morata.

Domènech, J. (2009). Elogi de l'educació lenta. Barcelona, Cataluña: Grao

Etxebarria, A. (2016). Iraunkortasunerako hezkuntza: Baso Eskolak (Trabajo de fin de Grado). UPV-EHU, Bilbao, País Vasco.

Feito, R. y López Ruiz, A. (2008). Construyendo escuelas democráticas. Barcelona, Cataluña: Hipatia.

Fukuyama, F. (1989). The end of history?. The national interest, (16), 3-18.

García, A. (2017). Otra educación ya es posible: Introducción a las pedagogías alternativas. Valencia, España: Litera.

García., T. (2005). El valor de la democracia en la escuela: Alternativas pedagógicas. Perspectiva Educacional, Formación de Profesores, (46), 27-42.

Giacomoni, V. (2008). La evolución del concepto de pedagogía libertaría: de la teoría a la práctica. Germina, 5, 85-97.

Greenberg, D. (2003). Por fin, libres. Educación democrática en Sudbury Valley School. Alicante, España: Marién Fuentes y Javier Herrero.

Guerrero, E. (2013). Sistema Amara Berri: 40 años de compromiso con la educación. Infancia: educar de 0 a 6 años, (139), 26-31

Martín, J. (2004). Paideia una escuela libertaria. Revista de Cultura, Asociacionismo y Movimientos Sociales, 34, 43-47. 
Mateu, J. (2011). Circunstancias, retos y posibilidades de las escuelas alternativas. Organización y gestión educativa, 4, 17-20.

Neill, A. S. (2009). Hijos en libertad. Barcelona, Cataluña: Gedisa.

Neill, A. S., y Torner, F. M. (1963).Summerhill: un punto de vista radical sobre la educación de los niños. México D.F, México: Fondo de Cultura Económica.

Nixon, C. (2015).Remembering why Forest Schools are Important: Nurturing Environmental Consciousness in the Early Years (Tesis Doctoral). McGill University Libraries.

Nuin, J. (2013). Visitar el león dormido: una experiencia de activación. Cuadernos de Pedagogía, 434, 53-58

Larrañeta, A. (2015, 5 de noviembre). Boom de la educación con pedagogías alternativas. 20 Minutos. Recuperado de https://www.20minutos. es/noticia/2450363/0/educacion/pedagogias-

Pericacho, F. J. (2014). Educación Social y modelos escolares alternativos: aproximación teórica, retos y posibilidades socio-educativas. RES, Revista de Educación Social, 18.

Ramos, S. Pericacho, F.J. y (2013). Sobre la renovación pedagógica y su enseñanza universitaria. Una propuesta metodológica. Cabás, 10, 143-168.

Raywid, M.A. (1994). Alternative Schools: The state of the art. Educational Leadership, 52(1), 26-31.

REEVO - Mapeo Colectivo de la Educación Alternativa. (2019). REEVO - Mapeo Colectivo de la Educación Alternativa. Recuperado de http://map.reevo.org/

Rodríguez, M. (2014). Dejadnos aprender. Reflexiones desde la pedagogía libertaria.Guadalajara, España: Volapük Ediciones.

Salmerón, S. (2010). Nuevas miradas educativas. Aportaciones de la educación social a las escuelas libres de Cataluña, Una alternativa profesional y pedagógica. VIII Jornadas de Redes de Investigación en Docencia Universitaria: nuevas titulaciones y cambio universitario. Universidad de Alicante.

Tonucci, F. (1996). La ciudad de los niños. Barcelona, Cataluña: Grao.

- (2014). La infancia es un sensible indicador ambiental de nuestras ciudades. Bizkaia Maitea. p.35

Wernicke, C. G. (1994). Educación holística y pedagogía Montessori. Educación Hoy, (10), 1-11.

Wild, R. (2012). Educar para ser: vivencias de una escuela activa. Barcelona, Cataluña: Herder Editorial.

(2013). Vivir y aprender de manera coherente, en un proceso de desarrollo sostenible. Cuadernos de Pedagogía, 434, 4852.

XELL-Xarxa d'Educació Lliure. (2018). Educacio Lliure. Barcelona, Cataluya. Recuperado de https://educaciolliure.org/es/nos-presentamos/ quienes-somos/ 